\title{
Improved Throughput Scaling in Wireless Ad Hoc Networks With Infrastructure
}

\author{
Won-Yong Shin $\dagger$, Sang-Woon Jeon†, Natasha Devroyeł, Mai H. Vuł, \\ Sae-Young Chung $\dagger$, Yong H. Lee $\dagger$, and Vahid Tarokh $\ddagger$ \\ $\lceil$ School of Electrical Engineering and Computer Science, KAIST, Daejeon 305-701, Korea \\ $\ddagger$ School of Engineering and Applied Sciences, Harvard University, Cambridge, MA 02138, USA \\ Email: wyshin@ stein.kaist.ac.kr; swjeon@kaist.ac.kr; ndevroye@ fas.harvard.edu; maivu@ seas.harvard.edu; \\ \{sychung, yohlee\}@ee.kaist.ac.kr; vahid@ seas.harvard.edu
}

\begin{abstract}
We analyze the benefits of infrastructure support in improving the throughput scaling in networks of $n$ randomly located wireless nodes. The infrastructure uses multi-antenna base stations (BSs), in which the number of BSs and the number of antennas at each BS can scale at arbtrary rates relative to $n$. We introduce two multi-antenna BS-based routing protocols and analyze their throughput scaling laws. Two conventional schemes not using BSs are also shown for comparison. In dense networks, we show that the BS-based routing schemes do not improve the throughput scaling. In contrast, in extended networks, we show what our BS-based routing schemes can, under certain network conditions, improve the throughput scaling significantly.
\end{abstract}

\section{INTRODUCTION}

In [1], Gupta and Kumar introduced and studied the throughput scaling in a large wireless ad hoc network. They showed that, using a multi-hop ( $\mathrm{MH})$ communication scheme in a network of $n$ source-destination (S-D) pairs randomly distributed in a unit area, the total throughput scales as $\Theta(\sqrt{n / \log n}) .{ }^{1}$ This scaling was improved to $\Theta(\sqrt{n})$ using percolation theory [3]. Recent results have shown that an almost linear throughput in the network, i.e., $\Theta\left(n^{1-\epsilon}\right)$ for an arbitrarily small $\epsilon>0$, is achievable by using a hierarchical cooperation (HC) strategy [4].

To further improve the throughput scaling, hybrid networks consisting of both wireless ad hoc nodes and infrastructure nodes, or equivalently base stations (BSs), were introduced and analyzed in [5]-[8]. BSs are assumed to be interconnected by high capacity wired links. It is strictly necessary for the number $m$ of BSs to exceed a threshold in order to obtain a linear throughput scaling in $m$.

In this paper, we analyze the throughput scaling laws for a more general hybrid network, in which there are $l$ antennas at each BS, allowing us to exploit the spatial dimension at each BS. ${ }^{2}$ By allowing the number $m$ of BSs and the number $l$ of antennas to scale at arbitrary rates relative to the number $n$ of wireless nodes, we derive the achievable rates as a

\footnotetext{
${ }^{1}$ We use the following notations: i) $f(x)=O(g(x))$ means that there exist constants $C$ and $c$ such that $f(x) \leq C g(x)$ for all $x>c$. ii) $f(x)=o(g(x))$ means $\lim _{x \rightarrow \infty} \frac{f(x)}{g(x)}=0$. iii) $f(x)=\Omega(g(x))$ if $g(x)=O(f(x))$. iv) $f(x)=$ $\omega(g(x))$ if $g(x)=o(f(x))$. v) $f(x)=\Theta(g(x))$ if $f(x)=O(g(x))$ and $g(x)=O(f(x))[2]$.

${ }^{2}$ When the carrier frequency is very high, it is possible to deploy many antennas at each BS since the wavelength is small.
}

function of these scaling parameters. We propose two routing protocols utilizing BSs. In the first protocol, multiple sources (nodes) transmit simultaneously to each BS using a direct single-hop multiple-access in the uplink and a direct singlehop broadcast from each BS in the downlink. In the second protocol, the high-speed BS links are combined with nearest neighbor routing via $\mathrm{MH}$ among the wireless nodes. We compare our results to two conventional schemes for strictly ad hoc networks: the MH protocol [1] and HC protocol [4].

We evaluate our proposed schemes in two different networks: dense networks of unit area, and extended networks of unit node density. In dense networks, we show that the presence of BSs does not improve the throughput scaling and $\mathrm{HC}$ always outperforms the other protocols. On the contrary, in extended networks, depending on the network configurations and the path-loss attenuation, our proposed BS-based protocols can improve the throughput scaling significantly. Part of the improvement comes from the multiple-access advantage over the conventional schemes: more antennas mean more transmit pairs that can be activated simultaneously (compared to those of the $\mathrm{MH}$ scheme), i.e., enough degree of freedom (DoF) gain is obtained, provided the number $m$ of BSs and the number $l$ of antennas per BS are large enough. In addition, the BSs help to reduce the per-hop distance, which leads to a larger received signal power than that of the HC scheme, thus allowing for a better throughput scaling in extended networks.

The rest of this paper is organized as follows. Section II describes our network model with infrastructure support. Our two BS-based protocols are characterized in Section III and their achievable throughput scalings for both dense and extended networks are analyzed in Section IV.

We refer to the full paper [9] for the detailed description and all the proofs.

\section{System Model}

We consider a two-dimensional wireless network that consists of $n$ S-D pairs uniformly and independently distributed on a torus having an area of one and $n$ in dense and extended networks, respectively. Suppose that the whole area is divided into $m$ square cells, each of which is covered by one BS with $l$ antennas at the center (see Fig. 1). It is assumed that no nodes are physically located inside the BSs. These parameters 


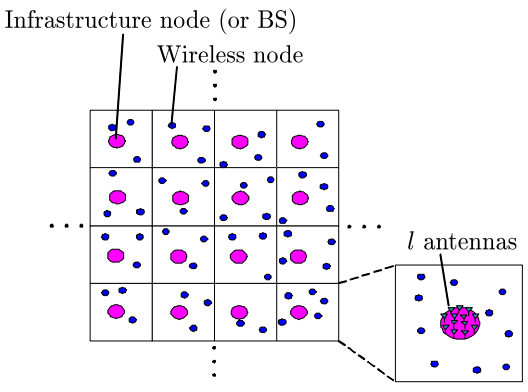

Fig. 1. The wireless ad hoc network with infrastructure support.

$n, m$, and $l$ are related according to

$$
n=m^{1 / \beta}=l^{1 / \gamma},
$$

where $\beta, \gamma \in[0,1)$. We allow the number $l$ of antennas to grow with the number of nodes and BSs in the network. The placement of these $l$ antennas depends on how the number of antennas scale as follows:

1) $l$ antennas are regularly placed on the BS boundary if $l=O(\sqrt{n / m})$,

2) $\sqrt{n / m}$ antennas are regularly placed on the BS boundary and the rest inside the boundary at equal spacing if $l=\omega(\sqrt{n / m})$ and $l=O(n / m)$, and

3) $l$ antennas are placed at equal spacing if $l=\omega(n / m){ }^{3}$ Furthermore, we assume that the BS-to-BS links have infinite capacity and these BSs are neither sources nor destinations. We suppose that the radius of each BS scales as $\epsilon_{0} / \sqrt{m}$ for dense networks and as $\epsilon_{0} \sqrt{n / m}$ for extended networks, where $\epsilon_{0}>0$ is an arbitrarily small constant. This radius scaling ensures the independence among the antennas.

We first describe the signal model in the uplink. Let $I \subset\{1, \cdots, n\}$ denote the set of simultaneously transmitting wireless nodes. Then, the $l \times 1$ received signal vector $\mathbf{y}_{s}$ at BS $s \in\{1, \cdots, m\}$ and the $l \times 1$ complex channel vector $\mathbf{h}_{s i}^{u}$ between node $i \in\{1, \cdots, n\}$ and BS $s$ are given by

$$
\mathbf{y}_{s}=\sum_{i \in I} \mathbf{h}_{s i}^{u} x_{i}+\mathbf{n}_{s}
$$

and

$$
\mathbf{h}_{s i}^{u}=\left[\frac{e^{j \theta_{s i, 1}^{u}}}{r_{s i, 1}^{u}{ }^{\alpha / 2}} \frac{e^{j \theta_{s i, 2}^{u}}}{r_{s i, 2}^{u}{ }^{\alpha / 2}} \cdots \frac{e^{j \theta_{s i, l}^{u}}}{r_{s i, l}^{u}{ }^{\alpha / 2}}\right]^{T},
$$

respectively, where $x_{i}$ is the transmit signal of the $i$-th node, and $\mathbf{n}_{s}$ denotes the complex additive white Gaussian noise (AWGN) vector whose element has zero-mean and variance $N_{0} . T$ denotes the transpose of a vector, and $\theta_{s i, t}^{u}$ is the random phase uniformly distributed over $[0,2 \pi]$ and independent for different $i, s, t$, and time (transmission symbol), i.e., fast fading. $r_{s i, t}^{u}$ and $\alpha>2$ denote the distance between node $i$ and the $t$-th antenna of BS $s$, and the path-loss exponent, respectively. The $1 \times l$ complex channel vector $\mathbf{h}_{i s}^{d}$ (in the downlink) between BS $s$ and node $i$, and the complex channel

\footnotetext{
${ }^{3}$ Such an antenna deployment guarantees both the nearest neighbor transmission from/to each BS and the enough space among the antennas.
}
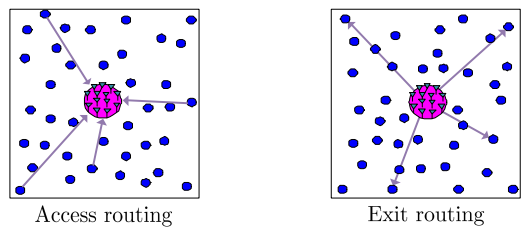

Fig. 2. The Infrastructure-supported single-hop (ISH) protocol.

$h_{k i}$ between nodes $i$ and $k$ can be modeled in a similar manner. Suppose that each node has an average transmit power constraint $P$ (constant). We assume that the average transmit power at each BS scales linearly with the number of nodes covered by one cell and each BS has the same per-antenna power constraint. Channel state information (CSI) is assumed to be available at the receivers but not at the transmitters (unless otherwise stated).

\section{Routing PROTOCOLS}

In this section, we present our two BS-based protocols. Two conventional schemes [1], [4] with no infrastructure support are also described.

\section{A. Protocol With Infrastructure Support}

We modify the conventional BS-based transmission schemes in [5]-[8] for our multi-antenna setting: a source node transmits its packet to the closest BS, the BS having the packet transmits it to the BS that is nearest to the destination via wired BS-to-BS links, and the destination finally receives its data from the nearest BS. Since there exist both access (to BSs) and exit (from BSs) routings, we use different time slots, e.g., even and odd time slots, respectively.

1) Infrastructure-supported single-hop (ISH) protocol: In contrast with previous works, we exploit the spatial dimensions enabled by having multiple antennas at each BS and, thus are able to support multiple transmission pairs simultaneously using a single BS. Now we propose an infrastructure-supported single-hop (ISH) transmission protocol shown in Fig. 2 under dense networks as the following:

- Divide the network into square cells of area $1 / m$ having one BS at their center.

- For the access routing, all source nodes in each cell, given by $n / m$ nodes with high probability (whp), transmit their independent packets simultaneously via single-hop multiple-access to the BS in the same cell. A transmit power of $\frac{P}{\max \{l, n / m\} m^{\alpha / 2}}$ is used at each uplink transmission.

- Each BS receives and jointly decodes packets from source nodes in the same cell and treats signals received from the other cells as noise. Each BS acts as a minimum mean-square error (MMSE) receiver [10] with successive interference cancellation (SIC), which is one of receive filters, in the uplink. More precisely, the $l \times 1$ unnormailzed receive filter $\mathbf{v}_{i}$ has the expression [10]

$$
\mathbf{v}_{i}=\left(\mathbf{I}_{l}+\sum_{k>i} \frac{P}{\max \left\{l, \frac{n}{m}\right\} m^{\alpha / 2}} \mathbf{h}_{s k}^{u} \mathbf{h}_{s k}^{u \dagger}\right)^{-1} \mathbf{h}_{s i}^{u},
$$


which means that the receiver of BS $s$ for the $i$-th node cancels signals from nodes $1, \cdots, i-1$ and treats signals from nodes $i+1, \cdots, n / m$ as noise, for every $i$, when we have the canceling order such as $1, \cdots, n / m$.

- For the exit routing, each BS transmits all received packets, i.e., $n / m$ packets whp, via single-hop broadcast to the destinations in the cell. The transmit filters in the downlink are the same as MMSE-SIC receive filters in the uplink. The CSI at the transmitter is only required at each BS to perform a transmit filtering in the downlink. A total transmit power of $\frac{P(n / m)}{\max \{l, n / m\} m^{\alpha / 2}}$ is used at each downlink transmission.

For the ISH protocol, more DoF gain is provided compared to transmissions via $\mathrm{MH}$. Note that when $\alpha>2$ the transmit power $\frac{P}{\max \{l, n / m\} m^{\alpha / 2}}$ at each node tends to zero as $n \rightarrow$ $\infty$. Hence, the given protocol satisfies the average power constraint $P$ in the uplink. Similarly, it is easily shown that the average power constraint at each BS is satisfied in the downlink.

For extended networks, we can apply the above protocol by converting the area of a cell to $n / m$, the required transmit power at each node to $\frac{P n^{\alpha / 2}}{\max \{l, n / m\} m^{\alpha / 2}}=$ $\frac{P}{\max \{l, n / m\}(m / n)^{\alpha / 2}}$, and the required transmit power at each BS to $\frac{(n / m) P n^{\alpha / 2}}{\max \{l, n / m\} m^{\alpha / 2}}=\frac{(n / m) P}{\max \{l, n / m\}(m / n)^{\alpha / 2}}$, respectively. However, since $\max \{l, n / m\}(m / n)^{\alpha / 2}=o(1)$ under most operating regimes, i.e., the network is power-limited, we violate the average power constraint if each node (or BS) transmits continuously. Instead of continuous transmissions, a bursty transmission scheme [4], which uses only a fraction

$$
\min \left\{1, \max \left\{l, \frac{n}{m}\right\}\left(\frac{m}{n}\right)^{\alpha / 2}\right\}
$$

of the time for actual transmission, can be used to meet the given average power constraint. Under the extended networks with ISH protocol, we may conclude that the total throughput decreases by $\min \left\{1, \max \{l, n / m\}(m / n)^{\alpha / 2}\right\}$ compared to the dense network scenario.

2) Infrastructure-supported multi-hop (IMH) protocol: To improve the throughput scaling of the extended (or powerlimited) network, we propose an infrastructure-supported multi-hop (IMH) transmission protocol in which multiple source nodes in a cell transmit their packets to its closet BS via $\mathrm{MH}$, thus having much higher received power than that of the direct one-hop transmission in extended networks. Similarly, each BS delivers its packets to the corresponding destinations by IMH transmissions. The proposed IMH transmission protocol in Fig. 3 under dense networks is as follows:

- Divide the network into square cells of area $1 / m$ each and again divide each cell into smaller square cells of area $2 \log n / n$ each, where we call these smaller cells routing cells.

- For the access routing, $\min \{l, \sqrt{n / m}\}$ source nodes in each cell transmit their independent packets using $\mathrm{MH}$ routing (which will be described in Section III-B) to the corresponding BS. Let us now consider how to set a $\mathrm{MH}$
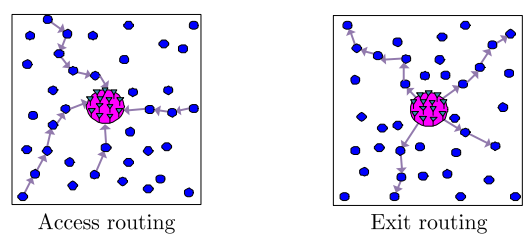

Fig. 3. The Infrastructure-supported multi-hop (IMH) protocol.

routing path from each source to the corresponding BS. We draw a line connecting a source to one antenna of its BS and perform $\mathrm{MH}$ routing horizontally or vertically by using the adjacent routing cells passing through the line until its packet reaches the corresponding receiver (antenna). Note that we can draw $\min \{l, \sqrt{n / m}\}$ lines such that there are no crossings in the cell. A transmit power $P / n^{\alpha / 2}$ at each node is used.

- We assume that each antenna placed only on the BS boundary receives its packet from the node of the nearest neighbor routing cell. If $l=\omega(\sqrt{n / m})$, each boundary routing cell in the BS has at least one BS antenna, and thus one of antennas inside the routing cell can receive a packet. Each receiver treats signals from the other nodes as noise, and decodes its packet independently.

- For the exit routing, we perform the MH routing from a BS to multiple destinations similar to the above access routing. From per-antenna power constraint at each BS, an average power at each BS antenna is constrained by $\frac{n P}{m l}$. One antenna in the routing cell of BS boundary transmits its packet to a destination via $\mathrm{MH}$ transmissions along a line connecting the antenna of its BS to the corresponding destination. We use a transmit power $P / n^{\alpha / 2}$ at each $\mathrm{BS}$ antenna (which satisfies the power constraint).

- Each routing cell operates based on 9-time division multiple access (TDMA) in which a routing cell transmits one of its allocated packets when it is activated to avoid a huge interference. We use a transmit power $P / n^{\alpha / 2}$ at each BS antenna.

Note that the node transmit power of the given protocol satisfies the average power constraint $P$ as $n$ goes to infinity. The per-antenna power constraint at each BS is also satisfied.

In a similar manner as the ISH protocol, by converting the area of a cell to square cells of area $n / m$ each and setting the area of a routing cell to $2 \log n$ and the required transmit power at each node/BS antenna to $P$, we can apply the IMH protocol to extended networks. However, it violates the average perantenna power constraint in the downlink for $l=\omega(n / m)$. In this case, all the antennas on the routing cell of BS boundary, i.e., $\frac{m l}{n}$ antennas, transmit same packets to one node of the nearest neighbor routing cell with a transmit power $\frac{n P}{m l}$. Then since our IMH protocol satisfies the power constraint, a total throughput does not decrease compared to the dense network case. 


\section{B. Protocols Without Infrastructure Support}

To improve throughput scalings of infrastructure-supported networks, the number of equipped BSs should be higher than a certain level. That is, pure ad hoc transmissions without help of the BSs may achieve better throughput scaling when the number $m$ of BSs is not large enough. We briefly introduce the $\mathrm{MH}$ protocol and the $\mathrm{HC}$ protocol which were proposed in [1] and [4], respectively.

1) $\mathrm{MH}$ protocol: The basic procedure of the $\mathrm{MH}$ protocol in dense networks is as follows:

- Divide the network into square routing cells of area $2 \log n / n$.

- Draw a line connecting a S-D pair. A source transmits a packet to its destination using the nodes in the adjacent routing cells passing through the line.

- A transmit power of $P / n^{\alpha / 2}$ is used.

- Each routing cell operates the $k$-TDMA to avoid large interference, where $k$ denotes some small constant independent of $n$.

For extended networks, we can apply the above protocol by converting the area of a cell to $2 \log n$ and the transmit power to $P$, respectively.

2) HC protocol: The $\mathrm{HC}$ consists of three phases as the following:

- Divide the network into clusters each having $M$ nodes.

- During the first phase, each source distributes its data to the other $M-1$ nodes in the same cluster.

- During the second phase, a long-range multiple-input multiple-output (MIMO) transmission between two clusters having a source and its destination is performed, one at a time.

- During the last phase, each node quantizes the received observations and delivers the quantized data to the corresponding destinations in the same cluster. By collecting all quantized observations, each destination can decode its packet.

When each node transmits data within its cluster, which is done during the first and third phases, we can apply another smaller-scaled cooperation within each cluster by dividing each cluster into smaller clusters. By continuing this procedure, it is possible to establish the hierarchical strategy in the network. A transmit power of $P / n$ is used in dense networks while the bursty HC scheme is performed in extended networks with the full power $P$ a fraction $1 / n^{\alpha / 2-1}$ of the time.

\section{ACHiEvable Throughrut SCALING}

In this section, we analyze the throughput scaling for both dense and extended networks under our routing protocols. Although the $\mathrm{HC}$ in [4] provides a near-optimal throughput scaling in dense networks, it may degrade throughput scalings in extended (or power-limited) networks. As a result, the best strategy among the four schemes ISH, IMH, MH, and HC depends on the path-loss exponent $\alpha$, the scaling parameters $\beta$ and $\gamma$ under extended networks.

\section{A. Dense Networks}

We first show the achievable rate of the ISH protocol in dense networks. The amount of interference in the ISH scheme is quantified as follows. Under our dense network model with ISH protocol, the total interference power at each BS antenna from simultaneously transmitting nodes in the uplink is upper-bounded by a constant. Each node also has a constant interference power from simultaneously transmitting BSs in the downlink. Thus, we get the following two results, which show the transmission rates for the access and exit routings, respectively. A rate of the access routing at each $\mathrm{BS}$ grows with $\Omega(\min \{l, n / m\})$. Note that $\min \{l, n / m\}$ corresponds to the DoF at each cell provided in the uplink of the ISH protocol. For the exist routing, we have the same rate as that of the access routing. Let $T_{n}$ denote the total throughput of the network. From the above observations, we obtain a lower bound on the capacity scaling $T_{n}$, and thus the following proposition presents the achievable rate of ISH protocol.

Proposition 1: When a dense network uses the ISH protocol,

$$
T_{n}=\Omega\left(m \min \left\{l, \frac{n}{m}\right\}\right)
$$

is achievable for all $m=n^{\beta}$ satisfying $\beta \in[0,1)$.

Next, we analyze the achievable rate of IMH protocol in dense networks. It is examined under the IMH protocol how many source nodes can be active simultaneously, maintaining a constant throughput $\Theta(1)$ per S-D pair.

Proposition 2: When a dense network uses the IMH protocol,

$$
T_{n}=\Omega\left(m \min \left\{l,\left(\frac{n}{m}\right)^{1 / 2-\epsilon}\right\}\right)
$$

is achievable for all $m=n^{\beta}$ satisfying $\beta \in[0,1)$, where $\epsilon>0$ is an arbitrarily small constant.

We now consider throughput scalings of two conventional protocols that do not utilize the BSs. The throughputs of the $\mathrm{MH}$ communication [1] and the HC scheme [4] are given by

$$
T_{n}=\Omega\left(n^{1 / 2-\epsilon}\right)
$$

and

$$
T_{n}=\Omega\left(n^{1-\epsilon}\right),
$$

for an arbitrarily small $\epsilon>0$, respectively. Based on the four achievability results, we may conclude the following. Suppose a dense network uses ISH, IMH, MH, and $\mathrm{HC}$ protocols. Then the HC, whose throughput scales almost linearly with $n$, outperforms the other protocols for any parameters $\alpha, \beta$, and $\gamma$. In other words, the throughput of ISH, IMH, and $\mathrm{MH}$ protocols cannot be beyond linear scaling for $\beta, \gamma \in[0,1)$, and $\alpha>2$. Hence, infrastructure does not improve the throughput scaling in dense networks.

\section{B. Extended Networks}

In the ISH protocol, the number of simultaneously transmitted sources in each cell is $n / m$, while only $\min \{l, \sqrt{n / m}\}$ sources can transmit simultaneously per cell in the IMH 


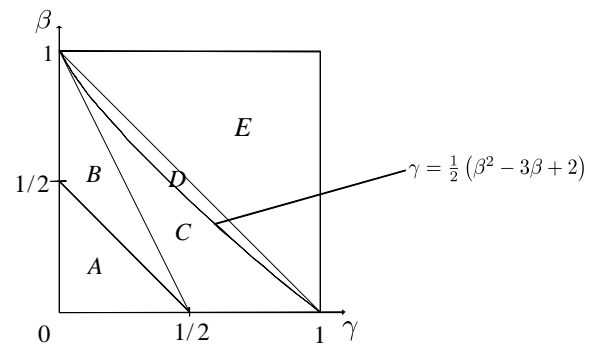

Fig. 4. Operating regimes on the achievable throughput scaling with respect to $\beta$ and $\gamma$.

protocol. The latter case, however, has an advantage over the former in terms of the shorter per-hop distance or higher received signal power, i.e., more power gain, in extended (or power-limited) networks. We show that the throughput scaling can be improved under some conditions by applying two BSbased transmissions in extended networks.

As stated in Section III, bursty transmission schemes are used to satisfy the power limitation in extended networks. Using the analysis similar to those used for dense networks, an achievable throughput of the ISH protocol is given as follows.

Proposition 3: When an extended network uses the ISH protocol,

$$
T_{n}=\Omega\left(m \min \left\{l, \frac{n}{m}\right\} \min \left\{1, \max \left\{l, \frac{n}{m}\right\}\left(\frac{m}{n}\right)^{\alpha / 2}\right\}\right)
$$

is achievable for all $m=n^{\beta}$ satisfying $\beta \in[0,1)$, where $\epsilon>0$ is an arbitrarily small constant.

If we perform the IMH protocol as in dense networks, we are in the power-limit for the case where $l=\omega(n / m)$ even if we transmit with full power $P$. Hence, as mentioned in Section III, our IMH scheme is slightly modified such that all antennas within the routing cell of BS boundary transmit same signals with a transmit power $\frac{n P}{m l}$. It also guarantees the signal-tointerference-and-noise ratio (SINR) $\Omega(1)$ at the receiver (node for the first hop from BS antennas), thus yielding the rate $\Omega(1)$ per S-D pair.

Proposition 4: When an extended network uses the IMH protocol,

$$
T_{n}=\Omega\left(m \min \left\{l,\left(\frac{n}{m}\right)^{1 / 2-\epsilon}\right\}\right)
$$

is achievable for all $m=n^{\beta}$ satisfying $\beta \in[0,1)$, where $\epsilon>0$ is an arbitrarily small constant.

Note that (11) is the same as that in the dense networks. From the results of [1], [4], we also get $T_{n}=\Omega\left(n^{1 / 2-\epsilon}\right)$ and $T_{n}=\Omega\left(n^{2-\alpha / 2-\epsilon}\right)$ for the $\mathrm{MH}$ and $\mathrm{HC}$ protocols, respectively.

From the achievable rates of each scheme, we obtain the following result. Suppose an extended network uses the four hybrid schemes ISH, IMH, MH, and HC. The best achievable scheme among those and its throughput scaling are shown in TABLE I according to the two-dimensional operating regimes with respect to $\beta$ and $\gamma$. Operating regimes $\mathrm{A}-\mathrm{E}$ on the
TABLE I

ACHIEVABLE RATES FOR AN EXTENDED NETWORK WITH INFRASTRUCTURE.

\begin{tabular}{c|c|c|c}
\hline Regime & Condition & Scheme & $T_{n}$ \\
\hline \hline \multirow{2}{*}{$\mathrm{A}$} & $2<\alpha<3$ & HC & $n^{2-\alpha / 2}$ \\
& $\alpha \geq 3$ & MH & $\sqrt{n}$ \\
\hline \multirow{2}{*}{$\mathrm{B}$} & $2<\alpha<4-2 \beta-2 \gamma$ & HC & $n^{2-\alpha / 2}$ \\
& $\alpha \geq 4-2 \beta-2 \gamma$ & IMH & $n^{\beta+\gamma}$ \\
\hline \multirow{2}{*}{$\mathrm{C}$} & $2<\alpha<3-\beta$ & HC & $n^{2-\alpha / 2}$ \\
& $\alpha \geq 3-\beta$ & IMH & $n^{(1+\beta) / 2}$ \\
\hline \multirow{2}{*}{$\mathrm{D}$} & $2<\alpha<\frac{2(1-\gamma)}{\beta}$ & HC & $n^{2-\alpha / 2}$ \\
& $\frac{2(1-\gamma)}{\beta} \leq \alpha<1+\frac{2 \gamma}{1-\beta}$ & ISH & $n^{1+\gamma-\alpha(1-\beta) / 2}$ \\
& $\alpha \geq 1+\frac{2 \gamma}{1-\beta}$ & IMH & $n^{(1+\beta) / 2}$ \\
\hline \multirow{2}{*}{$\mathrm{E}$} & $2<\alpha<\frac{2 \gamma}{1-\beta}$ & ISH & $n$ \\
& $\frac{2 \gamma}{1-\beta} \leq \alpha<1+\frac{2 \gamma}{1-\beta}$ & ISH & $n^{1+\gamma-\alpha(1-\beta) / 2}$ \\
& $\alpha \geq 1+\frac{2 \gamma}{1-\beta}$ & IMH & $n^{(1+\beta) / 2}$ \\
\hline
\end{tabular}

throughput scaling are shown in Fig. 4. It is important to verify which regime is feasible in realistic scenarios and the best protocol in different regimes. Regime $\mathrm{E}$ is less practical than the others, as the number $l$ of antennas scales faster than the number $n / m$ of nodes in a cell. Note that we can achieve a linear throughput for $2<\alpha<\frac{2 \gamma}{1-\beta}$ in Regime E. If we have smaller $m$ and $l$, we approach Regime A, in which the infrastructure is not helpful. In between these regimes, we observe some interesting regimes for which BSbased protocols are dominant. For example, Regime D has the following characteristics: the HC protocol has the highest throughput at low path-loss attenuation regime, but as the pathloss exponent $\alpha$ increases, the best scheme becomes the ISH protocol. This is because the penalty for long-range MIMO transmissions of the HC increases. Finally, the IMH protocol becomes dominant when $\alpha$ is large since the ISH protocol has the power limitation at high path-loss attenuation regime.

\section{REFERENCES}

[1] P. Gupta and P. R. Kumar, "The capacity of wireless networks," IEEE Trans. Inf. Theory, vol. 46, pp. 388-404, Mar. 2000.

[2] D. E. Knuth, "Big Omicron and big Omega and big Theta," ACM SIGACT News, vol. 8, pp. 18-24, Apr.-June 1976.

[3] M. Franceschetti, O. Dousse, D. N. C. Tse, and P. Thiran, "Closing the gap in the capacity of wireless networks via percolation theory," IEEE Trans. Inf. Theory, vol. 53, pp. 1009-1018, Mar. 2007.

[4] A. Özgür, O. Lévêque, and D. N. C. Tse, "Hierarchical cooperation achieves optimal capacity scaling in ad hoc networks," IEEE Trans. Inf. Theory, vol. 53, pp. 3549-3572, Oct. 2007.

[5] S. R. Kulkarni and P. Viswanath, "Throughput scaling for heterogeneous networks," in Proc. IEEE Int. Symp. Inform. Theory (ISIT), Yokohama, Japan, June/July 2003, p. 452.

[6] U. C. Kozat and L. Tassiulas, "Throughput capacity of random ad hoc networks with infrastructure support," in Proc. ACM MobiCom, San Diego, CA, Sep. 2003, pp. 55-65.

[7] A. Zemlianov and G. de Veciana, "Capacity of ad hoc wireless networks with infrastructure support," IEEE J. Select. Areas Commun., vol. 23, pp. 657-667, Mar. 2005.

[8] B. Liu, P. Thiran, and D. Towsley, "Capacity of a wireless ad hoc network with infrastructure," in Proc. ACM MobiHoc, Montréal, Canada, Sep. 2007.

[9] W.-Y. Shin, S.-W. Jeon, N. Devroye, M. H. Vu, S.-Y. Chung, Y. H. Lee, and V. Tarokh, "Improved capacity scaling in wireless networks with infrastructure," in preparation.

[10] P. Viswanath and D. N. C. Tse, "Sum capacity of the vector Gaussian broadcast channel and uplink-downlk duality," IEEE Trans. Inf. Theory, vol. 49, pp. 1912-1921, Aug. 2003. 